\title{
NONLINEAR WAVE EQUATIONS AND SINGULAR SOLUTIONS
}

\author{
HIDESHI YAMANE
}

(Communicated by David S. Tartakoff)

\begin{abstract}
We construct solutions to nonlinear wave equations that are singular along a prescribed noncharacteristic hypersurface, which is the graph of a function satisfying not the Eikonal but another partial differential equation of the first order. The method of Fuchsian reduction is employed.
\end{abstract}

\section{INTRODUCTION}

In our previous article [10, we constructed a solution to a nonlinear Cauchy problem and gave a lower bound of its lifespan.

In the present article, we consider certain nonlinear wave equations with polynomial nonlinearity. More precisely, the right-hand side of an equation in our class is a polynomial of degree 2 in the derivatives of the unknown function. Notice that some of these kinds of equations fall within the class treated in [10.

We construct solutions that are singular along a prescribed noncharacteristic hypersurface satisfying a certain condition written in terms of a partial differential equation of the first order. Blowup occurs due to the presence of a logarithmic term. Its coefficient (and hence the speed of the blowup) is determined by the hypersurface and the nonlinear term of the equation. On the other hand, it is possible to construct hypersurfaces in accordance with a given pair of the coefficient and the nonlinear term.

In later sections, we briefly discuss a nonlinear elliptic equation and multi-valued solutions to partial differential equations in the complex domain. We also consider equations with nonlinearity of higher order.

The proofs are based on Fuchsian reduction. This method, a powerful tool in many branches of analysis, was first employed by Kichenassamy and Littman in their construction of singular solutions to the equation $\square u=\exp u$ in [4]. Similar methods were used in [3], 7] and [8. We reduce our equations to Fuchsian ones and use results by Gérard and Tahara in [1] and 2] (Chapter 8). An alternative proof is the reduction to first-order Fuchsian systems, which are solved in [4] and [5].

Received by the editors March 21, 2006 and, in revised form, September 8, 2006.

2000 Mathematics Subject Classification. Primary 35L70, 35A20.

Key words and phrases. Nonlinear wave equation, nonlinear Fuchsian equation, singular Cauchy problem.

This research was partially supported by Grant-in-Aid for Scientific Research (No.17540182), Japan Society for the Promotion of Science.

(C)2007 American Mathematical Society Reverts to public domain 28 years from publication 


\section{MAIN RESUlt}

Let $f(t, x ; \tau, \xi)$ be a polynomial of degree 2 in $(\tau, \xi) \in \mathbb{R} \times \mathbb{R}^{n}$ whose coefficients are analytic functions in $(t, x)$ in an open set $U$ of $\mathbb{R} \times \mathbb{R}^{n}$. We decompose $f$ into homogeneous parts: $f=f_{2}+f_{1}+f_{0}$, where $f_{l}$ is homogeneous of degree $l$. For $l=1,2$, we sometimes denote $f_{l}(t, x ; \tau, \xi)$ by $f_{l}(\tau, \xi)$ for brevity. Notice that $f_{0}=f_{0}(t, x)$ is just a function in $(t, x)$ and is independent of $(\tau, \xi)$.

The primary purpose of the present paper is the study of a nonlinear wave equation

$$
u_{t t}-\Delta u=f\left(t, x ; \partial_{t} u, \nabla u\right)=\sum_{l=0}^{2} f_{l}\left(t, x ; \partial_{t} u, \nabla u\right),
$$

where $\nabla$ is the gradient with respect to $x$.

We construct a solution $u(t, x)$ which blows up on a prescribed noncharacteristic hypersurface. More precisely, our main result is the following:

Theorem 2.1. Let $\Sigma$ be a hypersurface defined by $\Sigma=\{(t, x) \in U: t=\psi(x)\}$, where $\psi(x)$ is an analytic function. Assume that

$$
0 \neq 1-|\nabla \psi(x)|^{2}=a f_{2}(\psi(x), x ;-1, \nabla \psi(x))
$$

in $U$ for a nonzero constant $a$. Then for any analytic function $v_{0}$ on $\Sigma$, there exists an analytic function $v(t, x)$ in an open neighborhood $\Omega$ of $\Sigma$ such that:

- $u(t, x)=-a \log (t-\psi(x))+v(t, x)$ is a solution to (2.1) in $\Omega \cap\{t>\psi(x)\}$,

- the restriction of $v$ on $\Sigma$ coincides with $v_{0}$.

Such a function $v(t, x)$ is unique in a neighborhood of $\Sigma$.

Remark 2.2. [A] If $f_{2}(\tau, \xi)=a^{-1}\left(\tau^{2}-|\xi|^{2}\right)$, then (2.2) reduces to $1-|\nabla \psi(x)|^{2} \neq 0$. It is satisfied by almost any function $\psi$.

[B] The function $\psi$ is a solution not to the eikonal equation but to another equation of the first order. It would be an interesting task to formulate a general theory encompassing the "pseudo-eikonal" equations (2.2), (8.2) and the curvature condition in [4. A promising approach is to deal with them in the framework of [6], but it is beyond the scope of the present paper.

[C] The speed of blowup is controlled by the coefficient $a$. It is calculated in terms of $f_{2}$ and $\psi$. On the other hand, we can find many $\psi$ 's satisfying (2.2) for a given pair $\left(f_{2}, a\right)$. We have only to use the Cauchy-Kovalevskaya theorem or the classical theory of partial differential equations of the first order.

Recall that the speed of blowup was always the same irrespective of $\psi$ in the study of $\square u=e^{u}$ in $[4]$ and $[5]$.

\section{Proof of Theorem 2.1}

We introduce a new system of coordinates $(T, X)$ by $T=t-\psi(x), X_{i}=x_{i}$ $(i=1,2, \ldots, n)$. The derivatives in the new variables are marked with ${ }^{\wedge}$ :

$$
\partial_{i}=\partial / \partial x_{i}, \partial_{t}=\partial / \partial t, \hat{\partial}_{i}=\partial / \partial X_{i}, \hat{\partial}_{X}=\left(\hat{\partial}_{1}, \ldots, \hat{\partial}_{n}\right), \hat{\partial}_{T}=\partial / \partial T .
$$

In the same way, $\Delta$ and $\nabla$ are the Laplacian and the gradient in $x$ respectively, while $\widehat{\Delta}$ and $\widehat{\nabla}$ are those in $X$. Subscripts on functions refer to the derivatives in $x$. For example, $\psi_{i}=\partial_{i} \psi, \psi_{i i}=\partial_{i}^{2} \psi$. 
The derivatives transform according to the following rules:

$$
\partial_{t}=\hat{\partial}_{T}, \quad \partial_{i}=-\psi_{i} \hat{\partial}_{T}+\hat{\partial}_{i} .
$$

We want to express the d'Alembertian $\square=\partial_{t}^{2}-\Delta$ in terms of $\hat{\partial}_{i}$ and $\hat{\partial}_{T}$. This calculation has already been explained in [4. We nevertheless repeat the argument here for the sake of the reader. It might seem a little confusing since it employs two systems of coordinates simultaneously. To begin with, we get

$$
\begin{aligned}
\partial_{i}^{2} & =\partial_{i}\left(-\psi_{i} \hat{\partial}_{T}+\hat{\partial}_{i}\right)=-\partial_{i}\left(\psi_{i} \hat{\partial}_{T}\right)+\partial_{i} \hat{\partial}_{i} \\
& =-\psi_{i} \partial_{i} \hat{\partial}_{T}-\psi_{i i} \hat{\partial}_{T}+\left(-\psi_{i} \hat{\partial}_{T}+\hat{\partial}_{i}\right) \hat{\partial}_{i} \\
& =-\psi_{i}\left(-\psi_{i} \hat{\partial}_{T}+\hat{\partial}_{i}\right) \hat{\partial}_{T}-\psi_{i i} \hat{\partial}_{T}-\psi_{i} \hat{\partial}_{T} \hat{\partial}_{i}+\hat{\partial}_{i}^{2} \\
& =\psi_{i}^{2} \hat{\partial}_{T}^{2}-2 \psi_{i} \hat{\partial}_{i} \hat{\partial}_{T}-\psi_{i i} \hat{\partial}_{T}+\hat{\partial}_{i}^{2} .
\end{aligned}
$$

Therefore $\square$ has the following form:

$$
\square=\Psi \hat{\partial}_{T}^{2}+2 \sum_{i=1}^{n} \psi_{i} \hat{\partial}_{i} \hat{\partial}_{T}+(\Delta \psi) \hat{\partial}_{T}-\widehat{\Delta},
$$

where $\Psi=\Psi(X)=\Psi(x)=1-|\nabla \psi(x)|^{2} \neq 0$.

Set $u(t, x)=-a \log T+v$, where $v$ is an unknown analytic function near $\Sigma=$ $\{T=0\}$. Then by (3.1), the left-hand side of (2.1) is

$$
\square u=\frac{a \Psi}{T^{2}}-\frac{a \Delta \psi}{T}+\square v .
$$

Next, let us calculate the right-hand side. We have

$$
\partial_{t} u=-a / T+\hat{\partial}_{T} v, \quad \partial_{i} u=a \psi_{i} / T-\psi_{i} \hat{\partial}_{T} v+\hat{\partial}_{i} v
$$

This leads to

$$
\begin{aligned}
\left(\partial_{t} u\right)^{2}= & \frac{a^{2}}{T^{2}}-\frac{2 a}{T} \hat{\partial}_{T} v+\left(\hat{\partial}_{T} v\right)^{2} \\
\left(\partial_{t} u\right)\left(\partial_{i} u\right)= & -\frac{a^{2} \psi_{i}}{T^{2}}+\frac{2 a \psi_{i}}{T} \hat{\partial}_{T} v-\frac{a}{T} \hat{\partial}_{i} v+\left(\hat{\partial}_{T} v\right)\left(-\psi_{i} \hat{\partial}_{T} v+\hat{\partial}_{i} v\right), \\
\left(\partial_{i} u\right)\left(\partial_{j} u\right)= & \frac{a^{2} \psi_{i} \psi_{j}}{T^{2}}+\frac{1}{T}\left\{-2 a \psi_{i} \psi_{j} \hat{\partial}_{T} v+a\left(\psi_{i} \hat{\partial}_{j}+\psi_{j} \hat{\partial}_{i}\right) v\right\} \\
& +\left(-\psi_{i} \hat{\partial}_{T} v+\hat{\partial}_{i} v\right)\left(-\psi_{j} \hat{\partial}_{T} v+\hat{\partial}_{j} v\right) .
\end{aligned}
$$

On the other hand, there exists an analytic function $\tilde{f}_{2}(T, X)$ in a neighborhood of $\Sigma$ such that

$$
f_{2}(t, x ;-1, \nabla \psi(x))=f_{2}(\psi(x), x ;-1, \nabla \psi(x))+T \tilde{f}_{2}(T, X) .
$$

We set $f_{2}(\Sigma)=f_{2}(\psi(x), x ;-1, \nabla \psi(x))$ for brevity. This quantity appears in (2.2) and should be distinguished from $f_{2}(-1, \nabla \psi(x))=f_{2}(t, x ;-1, \nabla \psi(x))$, a particular case of $f_{l}(\tau, \xi)=f_{l}(t, x ; \tau, \xi)$. We get

$$
\frac{f_{2}(-1, \nabla \psi(x))}{T^{2}}=\frac{f_{2}(\Sigma)}{T^{2}}+\frac{\tilde{f}_{2}(T, X)}{T} .
$$


Consequently, the right-hand side of equation (2.1) is

$$
\begin{aligned}
f\left(t, x ; \partial_{t} u, \nabla u\right)= & \frac{a^{2} f_{2}(\Sigma)}{T^{2}}+\frac{a^{2} \tilde{f}_{2}(T, X)}{T}-\frac{2 a}{T} f_{2}(-1, \nabla \psi) \hat{\partial}_{T} v \\
& +\frac{a}{T} L\left(T, X, \hat{\partial}_{X}\right) v+f_{2}\left(\hat{\partial}_{T} v,\left\{-\psi_{i} \hat{\partial}_{T} v+\hat{\partial}_{i} v\right\}_{i}\right) \\
& +\frac{a}{T} f_{1}(-1, \nabla \psi)+f_{1}\left(\hat{\partial}_{T} v,\left\{-\psi_{i} \hat{\partial}_{T} v+\hat{\partial}_{i} v\right\}_{i}\right)+f_{0}(t, x)
\end{aligned}
$$

where $L\left(T, X, \hat{\partial}_{X}\right)$ is a vector field with $[L, T]=0$.

Now let us compare (3.2) and (3.3). Our assumption (2.2) implies that the coefficients of $T^{-2}$ coincide: $a \Psi=a^{2} f_{2}(\Sigma)$. Therefore, in view of (3.1), the equation (2.1) is equivalent to the following one:

$$
\begin{aligned}
- & a \Delta \psi+T\left\{\Psi \hat{\partial}_{T}^{2} v+2 \sum_{i=1}^{n} \psi_{i} \hat{\partial}_{i} \hat{\partial}_{T} v+(\Delta \psi) \hat{\partial}_{T} v-\widehat{\Delta} v\right\} \\
= & a^{2} \tilde{f}_{2}(T, X)-2 \Psi \hat{\partial}_{T} v+a L\left(T, X, \hat{\partial}_{X}\right) v+T f_{2}\left(\hat{\partial}_{T} v,\left\{-\psi_{i} \hat{\partial}_{T} v+\hat{\partial}_{i} v\right\}_{i}\right) \\
& +a f_{1}(-1, \nabla \psi)+T f_{1}\left(\hat{\partial}_{T} v,\left\{-\psi_{i} \hat{\partial}_{T} v+\hat{\partial}_{i} v\right\}_{i}\right)+T f_{0}(t, x) .
\end{aligned}
$$

We divide this equation by $\Psi \neq 0$ and get an example of (4.1) in $\$ 4$ below. By Proposition 4.1] there exists a unique analytic solution $v$ to (3.4) with $\left.v\right|_{\Sigma}=v_{0}$ in a neighborhood of $\Sigma$. The function $u=-a \log T+v$ solves (2.1) in $\{T>0\}$. We have finally proved Theorem 2.1 .

\section{Nonlinear Fuchsian PARtial Differential EQUATIONS}

In this section, we prove a result on nonlinear Fuchsian partial differential equations which was used in the proof of Theorem 2.1.

We consider a nonlinear equation in a neighborhood of $\{0\} \times V \subset \mathbb{R}_{t} \times \mathbb{R}_{x}^{n}$, where $V$ is an open set of $\mathbb{R}_{x}^{n}$. We employ the usual notation for derivatives: $\partial_{i}=\partial / \partial x_{i}, \partial_{x}=\left(\partial_{1}, \ldots, \partial_{n}\right), \nabla v=\left(\partial_{1} v, \ldots, \partial_{n} v\right)$ and $\partial_{t}=\partial / \partial t$.

Now let us consider a Cauchy problem for the following nonlinear Fuchsian partial differential equation of weight 1 :

$$
\begin{aligned}
t \partial_{t}^{2} v+2 \partial_{t} v=\alpha & (t, x)+t P\left(t, x, \partial_{x}\right) \partial_{t} v+Q\left(t, x, \partial_{x}\right) v \\
& +t \beta(t, x)\left(\partial_{t} v\right)^{2}+R\left(t, x, \partial_{x}\right) v \cdot t \partial_{t} v+S(t, x ; \nabla v) .
\end{aligned}
$$

Here $\alpha$ and $\beta$ are analytic functions. The linear differential operators $P, Q$ and $R$ with analytic coefficients are of orders 1,2 and 1 , respectively, and $[P, t]=[Q, t]=$ $[R, t]=0$. We assume that $S(t, x ; \xi)$ is a quadratic form in $\xi$ whose coefficients are analytic functions in $(t, x)$.

Proposition 4.1. Let $v_{0}(x)$ be an analytic function in $V$. Then (4.1) has a unique analytic solution $v(t, x)$ with $v(0, x)=v_{0}(x)$ near $\{0\} \times V \subset \mathbb{R} \times \mathbb{R}^{n}$.

Proof. First we reduce the problem to the case $v_{0}(x) \equiv 0$. We introduce a new unknown function $w$ by $v(t, x)=v_{0}(x)+w(t, x)$. Then we have $t \partial_{t}^{2} v+2 \partial_{t} v=$ $t \partial_{t}^{2} w+2 \partial_{t} w$ 
We denote the right-hand side of (4.1) by $N[v]$. An elementary computation shows that

$$
\begin{aligned}
N[v] & =N\left[v_{0}+w\right] \\
& =N[w]+Q v_{0}+R v_{0} \cdot t \partial_{t} w+S\left(t, x ; \nabla v_{0}\right)+T(t, x ; \nabla w),
\end{aligned}
$$

where $T(t, x ; \xi)=\sum_{i=1}^{n} c_{i}(t, x) \xi_{i}$ for some analytic function $c_{i}(t, x)$. This implies that $w(t, x)$ satisfies an equation of the type (4.1), possibly with different coefficients. Therefore we have only to prove that (4.1) has a unique analytic solution $v(t, x)$ with $v(0, x) \equiv 0$. Multiplication by $t$ yields a nonlinear Fuchsian partial differential equation, solved in [1] and [2]. Its characteristic polynomial is $\rho^{2}+\rho$, and there exists a unique analytic solution $v(t, x)$ with $v(0, x) \equiv 0$.

Remark 4.2. [A] There is a stronger uniqueness result due to Gérard and Tahara. We only give a sketch here and refer the reader to [1] or [2] for a precise statement.

Assume that $v_{0}(x) \equiv 0$ for simplicity and let $\tilde{v}(t, x)$ be a multi-valued holomorphic solution to (4.1) in the universal covering space of $\{t \neq 0\} \subset \mathbb{C}_{t} \times \mathbb{C}_{x}^{n}$. If there exists a positive constant $p$ such that $\tilde{v}(t, x)=O\left(|t|^{p}\right)$ as $t$ tends to zero, then $\tilde{v}(t, x)$ is nothing but the solution $v(t, x)$ in Proposition 4.1

This observation enables us to improve the uniqueness part of Theorem 2.1, It rules out some functions involving terms of the form $T^{\alpha} \log ^{\beta} T, \alpha>0, T=t-\psi(x)$.

[B] The present formulation, a very restrictive one, has been chosen because it is all we need in the proof of Theorem 2.1. There is much room for generalization. We can easily consider characteristic polynomials other than $\rho^{2}+\rho$. Difficulties arise if we consider nonlinear terms that are not entire in their arguments; it is not always possible to consider large initial values.

\section{Time-Reversal}

In this section we discuss time-reversal and construct solutions on the negative side of $\Sigma$ under certain conditions. The equation (2.1) is not invariant under timereversal. Indeed, if we set $s=-t$, it becomes

$$
u_{s s}-\Delta u=f\left(-s, x,-\partial_{s} u, \nabla u\right) .
$$

In addition to (2.2), we assume

$$
f_{2}(t, x ; 1, \nabla \psi)=f_{2}(t, x ;-1, \nabla \psi) .
$$

Here notice that $f_{2}(\tau, \xi)=f_{2}(-\tau,-\xi)$ is trivial. The condition (5.2) is satisfied if $f_{2}(\tau, \xi)$ is free of terms of the form $c_{j}(t, x) \tau \xi_{j}$.

We set $\tilde{\psi}(x)=-\psi(x)$ and introduce $\tilde{f}_{2}(\tau, \xi)=f_{2}(-\tau, \xi)$, which is, to (5.1), what $f_{2}$ is to (2.1). Combining (2.2) and (5.2) yields

$$
0 \neq 1-|\nabla \tilde{\psi}(x)|^{2}=a \tilde{f}_{2}(-1, \nabla \tilde{\psi}(x)) .
$$

By using Theorem 2.1, we can find solutions to (5.1) in $\{(s, x): s>\tilde{\psi}(x)\}$. This gives a solution to (2.1) in $\{(t, x): t<\psi(x)\}$, the negative side of $\Sigma$.

To sum up, under (2.2) and (5.2), there exist solutions defined on both sides of the noncharacteristic hypersurface $\Sigma$ and their singularities propagate along it. 


\section{ElLiptic CASE}

We can apply a similar argument to

$$
\Delta u=a^{-1}|\nabla u|^{2}
$$

in $\mathbb{R}^{n}$, where $a$ is a nonzero constant. In this case, we can treat any analytic nonsingular hypersurface $\Lambda$ at least locally. Indeed, after rotation, it can be written in the form $\Lambda=\left\{x_{1}=\phi\left(x_{2}, \ldots, x_{n}\right)\right\}$. Notice that the equation is rotation-invariant. The function $1+\sum_{i=2}^{n}\left(\partial \phi / \partial x_{i}\right)^{2}>0$ replaces $\Psi$. Hence we have the following:

Theorem 6.1. Let $p$ be a point of an arbitrary analytic nonsingular hypersurface

$\Lambda$. Then there exists a neighborhood $U$ of $p$ such that in a connected component of $U \backslash \Lambda$ there exists an analytic solution $u(x)$ to (6.1) which blows up along $\Lambda \cap U$.

\section{Multi-Valued SOlutions in the COMPlex DOMain}

Our calculus is valid in the complex domain and the solution $u(t, x)$ in Theorem 2.1 becomes a multi-valued holomorphic function around the noncharacteristic complex hypersurface $\Sigma^{\mathbb{C}}=\left\{(t, x) \in \mathbb{C}^{n+1}: t=\psi(x)\right\}$. It is the unique solution to the following singular Cauchy problem near a point $\left(t_{0}, p\right) \in \Sigma^{\mathbb{C}}, t_{0}=\psi(p)$ :

$$
\left\{\begin{array}{l}
\square u=f\left(t, x, \partial_{t} u, \nabla u\right), \\
u\left(t_{0}, x\right)=-a \log \left(t_{0}-\psi(x)\right)+v\left(t_{0}, x\right), \\
\partial_{t} u\left(t_{0}, x\right)=-a /\left(t_{0}-\psi(x)\right)+\partial_{t} v\left(t_{0}, x\right) .
\end{array}\right.
$$

For such Cauchy data, singularities propagate along the noncharacteristic hypersurface $\Sigma^{\mathbb{C}}$ and only along it. Notice that some results in [4] and [5] are open to similar interpretations.

Several authors have constructed multi-valued solutions to nonlinear equations around characteristic hypersurfaces. See [1, 2, [5] and other works by Kichenassamy and Tahara concerning Fuchsian problems. They give solutions which are singular along the initial surface $\{t=0\}$. Moreover, see [9] and the references cited therein for singularities obtained by solving eikonal equations.

\section{EQUATIONS WITH NONLINEARITY OF HIGHER ORDER}

The leading term of a singular solution can be guessed by solving an ordinary differential equation. For example, one finds a solution $u(t)=\log 2-2 \log t$ to $d^{2} u / d t^{2}=e^{t}$ and it was the basis of [4] and [5]. On the other hand, the solution $u(t)=-\log t$ to $d^{2} u / d t^{2}=(d u / d t)^{2}$ is the simplest case of our Theorem 2.1.

We are naturally tempted to solve $d^{2} u / d t^{2}=-(d u / d t)^{m+1}$ for $m \geq 2$ and get $u(t)=C_{m} t^{(m-1) / m}$, where $C_{m}=m^{(m-1) / m} /(m-1)$. This solution vanishes as $t>0$ tends to 0 , but its derivative blows up. In this section, we give a multidimensional version of this fact.

Let $f(t, x ; \tau, \xi)$ be a polynomial of degree $m+1(m \geq 2)$ in $(\tau, \xi) \in \mathbb{R} \times \mathbb{R}^{n}$ whose coefficients are analytic functions in $(t, x)$ in an open set $U$ of $\mathbb{R} \times \mathbb{R}^{n}$. We decompose $f$ into homogeneous parts: $f=\sum_{l=0}^{m+1} f_{l}$, where $f_{l}$ is homogeneous of degree $l$. We sometimes denote $f_{l}(t, x ; \tau, \xi)$ by $f_{l}(\tau, \xi)$ for short.

We consider

$$
u_{t t}-\Delta u=f\left(t, x ; \partial_{t} u, \nabla u\right)=\sum_{l=0}^{m+1} f_{l}\left(t, x ; \partial_{t} u, \nabla u\right)
$$


and construct a solution $u(t, x)$ which vanishes on a prescribed noncharacteristic hypersurface.

Theorem 8.1. Let $\Sigma$ be a hypersurface defined by $\Sigma=\{(t, x) \in U: t=\psi(x)\}$. Assume

$$
\begin{aligned}
& 0 \neq 1-|\nabla \psi(x)|^{2}=\frac{(-m+1)^{m} a^{m}}{m^{m-1}} f_{m+1}(\psi(x), x ;-1, \nabla \psi(x)), \\
& f_{m}(\psi(x), x ;-1, \nabla \psi(x))=0
\end{aligned}
$$

for a nonzero constant $a$. Then there exists an open neighborhood $\Omega$ of $\Sigma$ and an analytic solution $u(t, x)$ to (8.1) in $\Omega \cap\{t>\psi(x)\}$ such that:

$$
\begin{aligned}
u(t, x) & \sim a(t-\psi(x))^{(m-1) / m} \text { (vanishing), } \\
\partial_{t} u(t, x) & \sim a \frac{m-1}{m}(t-\psi(x))^{-1 / m} \quad \text { (blow-up) }
\end{aligned}
$$

as $t-\psi(x) \rightarrow+0$. This solution $u(t, x)$ is analytic in $\left((t-\psi)^{1 / m}, x\right)$ and no logarithm is involved.

Remark 8.2. The conditions (8.2) and (8.3) are equivalent to the existence of such analytic functions $\tilde{f}_{m+1}(T, X)$ and $\tilde{f}_{m}(T, X)$ that

(8.2) $f_{m+1}(t, x ; 1,-\nabla \psi(x))=\frac{m^{m-1}}{(-m+1)^{m} a^{m}}\left(1-|\nabla \psi(x)|^{2}\right)+T \tilde{f}_{m+1}(T, X)$,

(8.3) $f_{m}(t, x ; 1,-\nabla \psi(x))=T \tilde{f}_{m}(T, X)$.

Proof. We introduce a system of coordinates $(T, X)$ as before and seek a solution $u$ of the form

$$
u=a T^{(m-1) / m}+T v
$$

where $v$ is an unknown function analytic in $\left(T^{1 / m}, X\right)$. It may have fractional-power singularities as a function in $T$. By using the formulas

$$
\hat{\partial}_{T} T=T \hat{\partial}_{T}+1, \quad \hat{\partial}_{T}^{2} T=T \hat{\partial}_{T}^{2}+2 \hat{\partial}_{T}
$$

and (3.1), we can express the left-hand side of (8.1) as follows:

$$
\begin{aligned}
\square u= & L_{1}+a \frac{m-1}{m}(\Delta \psi) T^{-1 / m} \\
& +\left\{\Psi\left(T \hat{\partial}_{T}^{2}+2 \hat{\partial}_{T}\right)+2 \sum_{i=1}^{n} \psi_{i}\left(T \hat{\partial}_{T} \hat{\partial}_{i}+\hat{\partial}_{i}\right)+(\Delta \psi)\left(T \hat{\partial}_{T}+1\right)-T \widehat{\Delta}\right\} v
\end{aligned}
$$

where $L_{1}=a \frac{-(m-1)}{m^{2}} \Psi T^{-(m+1) / m}$.

Next we shall compute the right-hand side of (8.1). We get

$$
\left\{\begin{aligned}
\partial_{t} u & =a \frac{m-1}{m} T^{-1 / m}+\left(T \hat{\partial}_{T} v+v\right), \\
\partial_{i} u & =-\psi_{i} \partial_{t} u+T \hat{\partial}_{i} v .
\end{aligned}\right.
$$

Notice that the expression of $\partial_{i} u$ contains $\partial_{t} u$. The latter quantity plays an important role in the following calculation. We have

$$
\begin{aligned}
f_{l}\left(\partial_{t} u, \nabla u\right) & =f_{l}\left(\partial_{t} u,\left\{-\psi_{i} \partial_{t} u+T \hat{\partial}_{i} v\right\}_{i}\right) \\
& =f_{l}(1,-\nabla \psi)\left(\partial_{t} u\right)^{l}+R_{l},
\end{aligned}
$$


where $R_{l}$ is a homogeneous polynomial of degree $l$ in $\partial_{t} u$ and $T \hat{\partial}_{i} v$ and is of degree $\leq \max \{0, l-1\}(\leq m)$ with respect to $\partial_{t} u$. It is of degree $\geq 1$ with respect to $T \hat{\partial}_{i} v$ if $l \geq 1$. The "spare" factor $T$ in $T \hat{\partial}_{i} v$ helps us since we are trying to get a Fuchsian equation; it offsets the negative power of $T$ in $\partial_{t} u$.

We have

$$
\left(\partial_{t} u\right)^{l}=\left(a \frac{m-1}{m}\right)^{l} T^{-l / m}+l\left(a \frac{m-1}{m}\right)^{l-1} T^{-(l-1) / m}\left(T \hat{\partial}_{T} v+v\right)+B_{l},
$$

where $B_{l}$ is the remainder of the binomial expansion, that is,

$$
B_{l}=\sum_{j=0}^{l-2}\left(\begin{array}{l}
l \\
j
\end{array}\right)\left(a \frac{m-1}{m}\right)^{j} T^{-j / m}\left(T \hat{\partial}_{T} v+v\right)^{l-j} .
$$

Since $f=\sum_{l=0}^{m+1} f_{l}$, we have by (8.6) and (8.7),

$$
f\left(\partial_{t} u, \nabla u\right)=\left\{L_{2}+L_{3}+f_{m+1}(1,-\nabla \psi) B_{m+1}\right\}+\sum_{l=0}^{m+1} R_{l}+\sum_{l=0}^{m} f_{l}(1,-\nabla \psi)\left(\partial_{t} u\right)^{l},
$$

where

$$
\begin{aligned}
& L_{2}=f_{m+1}(1,-\nabla \psi(x))\left(a \frac{m-1}{m}\right)^{m+1} T^{-(m+1) / m}, \\
& L_{3}=f_{m+1}(1,-\nabla \psi(x)) \cdot(m+1)\left(a \frac{m-1}{m}\right)^{m} T^{-1}\left(T \hat{\partial}_{T} v+v\right) .
\end{aligned}
$$

We set

$$
L_{2}^{\prime}=f_{m+1}(\psi(x), x ; 1,-\nabla \psi(x))\left(a \frac{m-1}{m}\right)^{m+1} T^{-(m+1) / m} .
$$

Then there exists an analytic function $L_{2}^{\prime \prime}(T, X)$ such that

$$
L_{2}=L_{2}^{\prime}+T^{-1 / m} L_{2}^{\prime \prime}(T, X) .
$$

The assumption (8.2) implies that $L_{1}=L_{2}^{\prime}$ and that

$$
L_{3}=-\Psi \cdot \frac{m+1}{m} T^{-1}\left(T \hat{\partial}_{T} v+v\right) .
$$

Note that (8.2) is written in terms of $(-1, \nabla \psi)$ instead of $(1,-\nabla \psi)$. By subtracting $L_{1}=L_{2}^{\prime}$ from (8.1), we come very close to a Fuchsian equation in view of (8.5) and (8.8).

We set $s=T^{1 / m}, \hat{\partial}_{s}=\partial / \partial s$ in $T>0$. Since $m T \hat{\partial}_{T}=s \hat{\partial}_{s}$, we have

$$
\begin{aligned}
T \hat{\partial}_{T} v+v & =\frac{1}{m}\left(s \hat{\partial}_{s} v+m v\right), \\
\partial_{t} u & =\frac{1}{m} s^{-1}\left\{a(m-1)+s\left(s \hat{\partial}_{s} v+m v\right)\right\} .
\end{aligned}
$$

Now we multiply the equation $\square u-L_{1}=f-L_{2}^{\prime}$ by $m^{2} s^{m} / \Psi$ and obtain a nonlinear Fuchsian equation which belongs to the class treated in [1] and [2]. Namely it has 
the form:

$$
\begin{aligned}
& \frac{a m(m-1)}{\Psi}(\Delta \psi) s^{m-1}+\left\{\left(s \hat{\partial}_{s}\right)^{2}+m s \hat{\partial}_{s}\right\} v \\
& +s^{m} P_{1}\left(s, X ;\left\{\left(s \hat{\partial}_{s}+m\right) \hat{\partial}_{i} v\right\}_{i}, s \hat{\partial}_{s} v+m v, \widehat{\Delta} v\right) \\
=s^{m-1} & \tilde{L}_{2}(s, X)-(m+1)\left(s \hat{\partial}_{s} v+m v\right)+s P_{2}\left(s, X ; s \hat{\partial}_{s} v+m v\right)\left(s \hat{\partial}_{s} v+m v\right)^{2} \\
& +s^{m} P_{3}\left(s, X ; s \hat{\partial}_{s} v+m v,\left\{\hat{\partial}_{i} v\right\}_{i}\right) \\
& +\sum_{l=0}^{m} Q_{l}(s, X) s^{\max \{m-l, 1\}}\left\{a(m-1)+s\left(s \hat{\partial}_{s} v+m v\right)\right\}
\end{aligned}
$$

Here $\tilde{L}_{2}(s, X)$ is an analytic function and $P_{1}, P_{2}$ and $P_{3}$ are polynomials in the arguments coming after the semicolons. Their coefficients and $Q_{l}$ are analytic functions in $(s, X)$ near $s=0$. The exponent of $s$ immediately after $Q_{l}$ is not $m-l$ but $\max \{m-l, 1\}$ because of (8.3). The characteristic polynomial is $(\rho+m)(\rho+m+1)$, and there exists a solution $v$, which is an analytic function in $(s, X)=\left((t-\psi)^{1 / m}, x\right)$.

\section{REFERENCES}

[1] Gérard R. and Tahara H., Solutions holomorphes et singulières d'équations aux dérivées partielles singulières non linéaires, Publ. Res. Inst. Math. Sci., 29(1993), 121-151. MR1208031 (94h:35005)

[2] Gérard R. and Tahara H., "Singular nonlinear partial differential equations", Vieweg, Bonn. (1996) MR 1757086 (2001c:35056)

[3] Kobayashi T., Singular solutions and prolongation of holomorphic solutions to nonlinear differential equations, Publ. RIMS, Kyoto Univ., 34(1998), 43-63. MR1617617 (99c:35004)

[4] Kichenassamy S. and Littman W., Blow-up surfaces for nonlinear wave equations, I, Comm. Partial Differential Equations, 18(3\&4)(1993), 431-452. MR1214867(94f:35087)

[5] Kichenassamy S. and Littman W., Blow-up surfaces for nonlinear wave equations, II, Comm. Partial Differential Equations, 18(11)(1993), 1869-1899. MR.1243529 (94m:35197)

[6] Kichenassamy S. and Srinivasan G. K., The structure of WTC expansions and applications, J. Phys. A: Math. Gen., 28(1995), 1977-2004. MR1336507 (96c:35029)

[7] Tahara H., On the singularities of solutions of nonlinear partial differential equations in the complex domain, "Microlocal Analysis and Complex Fourier Analysis", World Sci. Publ., Hackensack, NJ (2002), 273-283. MR2068543 (2005b:35005)

[8] Tahara H., On the singularities of solutions of nonlinear partial differential equations in the complex domain, II, "Differential equations \& asymptotic theory in mathematical physics", Ser. Anal. 2, World Sci. Publ., Hackensack, NJ (2004), 343-354. MR2161979 (2006d:35030)

[9] Uchikoshi K., Singular Cauchy problems for quasilinear equations of order two, J. Math. Pures Appl. (9) 83(9)(2004), 1151-1178. MR2091958 (2005k:35006)

[10] Yamane H., Nonlinear Cauchy problems with small analytic data, Proc. Amer. Math. Soc. 134(11)(2006), 3353-3361. MR2231920

Department of Physics, Kwansei Gakuin University, Gakuen 2-1, Sanda, Hyougo 6691337, JAPAN

E-mail address: yamane@ksc.kwansei.ac.jp; yamanehideshi@95.alumni.u-tokyo.ac.jp 